\title{
Novos alvos farmacológicos contra a COVID-19: o uso de Interferons para a ativação do estado antiviral e eliminação do vírus SARC-CoV-2
}

\author{
New pharmacological targets against COVID-19: the use of interferons for activating the antiviral \\ state and eliminating the SARC-CoV-2 virus \\ Nuevos objetivos farmacológicos contra COVID-19: el uso de interferones para la activación del \\ estado antiviral y la eliminación del virus SARC-CoV-2
}

Recebido: 17/08/2021 | Revisado: 22/08/2021 | Aceito: 26/08/2021 | Publicado: 29/08/2021

Lucas Mainardo Rodrigues Bezerra

ORCID: https://orcid.org/0000-0003-3659-3066 Faculdade de Ciências Humanas, Exatas e da Saúde do Piaú, Brasil E-mail: lucasmainardo@hotmail.com

Pedro Oliveira Carvalho Neto

ORCID: https://orcid.org/0000-0003-0428-7179 Faculdade de Ciências Humanas, Exatas e da Saúde do Piauí, Brasil E-mail: c.pedrooliveira@gmail.com

Fernando José de Morais Silva

ORCID: https://orcid.org/0000-0002-2224-8328 Faculdade de Ciências Humanas, Exatas e da Saúde do Piauí, Brasil E-mail: fernandojose.vdc13@gmail.com

Madelyne Alice Brito Ramos

ORCID: https://orcid.org/0000-0001-9155-3150 Faculdade de Ciências Humanas, Exatas e da Saúde do Piauí, Brasil E-mail: madelynealice@ hotmail.com

Ana Maria Santos Cardoso

ORCID: https://orcid.org/0000-0001-7092-2295 Faculdade de Ciências Humanas, Exatas e da Saúde do Piauí, Brasil E-mail: anamariacardoso05@gmail.com

Elisiel Martins de Sousa

ORCID: https://orcid.org/0000-0001-5752-1139 Faculdade de Ciências Humanas, Exatas e da Saúde do Piauí, Brasil E-mail: elisielmartinsdesousa@gmail.com

Moacir Ximenes Sousa Neto

ORCID: https://orcid.org/0000-0003-0324-9648

Centro Universitário Uninovafapi, Brasil

E-mail: moacirximenes@gmail.com

Tássia Layane Pontes Alencar

ORCID: https://orcid.org/0000-0002-4161-3589

Faculdade de Ciências Humanas, Exatas e da Saúde do Piauí, Brasil

E-mail: tassiapontesalencar@gmail.com

Luan Kelves Miranda de Souza

ORCID: https://orcid.org/0000-0002-8019-4022

Faculdade de Ciências Humanas, Exatas e da Saúde do Piauí, Brasil

Universidade Federal do Piauí, Brasil

E-mail: luan.souza@iesvap.edu.br

\begin{abstract}
Resumo
O vírus SARS-CoV-2, causador da doença COVID-19, foi descrito pela primeira vez em dezembro de 2019, na cidade de Wuhan, China, infectando mais de 170 milhões de pessoas. No decorrer da infecção viral, o sistema imunológico inato atua inicialmente com a sinalização celular levando a produção e liberação de citocinas próinflamatórias, caracterizadas na maioria das vezes por baixos níveis de interferons (IFNs), que estimulam a expressão de genes que contribuem para o deslocamento das células hospedeiras em direção a um estado antiviral. No decurso da SARS-CoV-2, a expressão e, as funções dos IFN-I são relativamente suprimidas. Este estudo tem como objetivo avaliar o potencial terapêutico do uso do Interferon na ativação do estado antiviral, principalmente nas fases iniciais da doença. Trata-se de uma revisão integrativa da literatura, utilizando-se de uma metodologia exploratória e descritiva. Os dados foram organizados de forma a elencar os estudos relacionados à temática em questão, a filtragem dos artigos encontrados teve como critério de inclusão, artigos nos anos de 2020 e 2021(com exceção de um deles, no ano de 2015), nos idiomas: português, inglês e espanhol. Considerando que o SARS-CoV-2 suprime a expressão do IFN-I e os genes induzidos por IFN, interrompendo os mecanismos de defesa imunológica, nota-se relação da
\end{abstract}


supressão dos IFN com o agravamento do quadro clínico em pacientes com COVID-19. Com isso, a literatura elucida que a administração exógena de IFN-I mostra reduzir a gravidade dos sintomas, porém, esse mecanismo precisa ser analisado com maior clareza.

Palavras-chave: Antivirais; Coronavírus; Interferons.

\begin{abstract}
SARS-CoV-2 virus, cause of COVID-19 disease, was described at first in Wuhan (China) December 2019, infecting more than 170 million people. During viral infection, innate immune system initially acts with cell signaling leading to the production and release of pro-inflammatory cytokines, mostly characterized by low levels of interferons (IFNs), which stimulate the expression of genes that contribute to the displacement of host cells towards an antiviral state. In the SARS-CoV-2, the expression and functions of IFN-I are relatively suppressed. This study aims to evaluate the therapeutic potential of using Interferon in activating the antiviral state, especially in the early stages of the disease. This is an integrative literature review, using an exploratory and descriptive methodology. The data were organized in order to list the studies related to the theme in question, the filtering of the articles found had as inclusion criteria, articles in the years 2020 and 2021 (except for one of them, in the year of 2015), in the languages: Portuguese, English and Spanish. Considering that SARS-CoV-2 suppresses the expression of IFN-I and the genes induced by IFN, interrupting the immune defense mechanisms, there is a relationship of IFN suppression with the worsening of the clinical picture in patients with COVID-19. So that, literature elucidates that the exogenous administration of IFNI has been shown to reduce the severity of symptoms, however, this mechanism needs to be analyzed more clearly.
\end{abstract}

Keywords: Antiviral agents; Coronavirus; Interferons.

\title{
Resumen
}

El virus SARS-CoV-2, que causa la enfermedad COVID-19, se describió por primera vez en diciembre de 2019 en la ciudad de Wuhan, China, infectando a más de 170 millones de personas. Durante la infección viral, el sistema inmunológico innato actúa inicialmente con la señalización celular que conduce a la producción y liberación de citocinas proinflamatorias, caracterizadas principalmente por niveles bajos de interferones (IFN), que estimulan la expresión de genes que contribuyen al desplazamiento de las células huésped. hacia un estado antiviral. Durante el SARS-CoV-2, la expresión y funciones de IFN-I están relativamente suprimidas. Este estudio tiene como objetivo evaluar el potencial terapéutico del uso de interferón para activar el estado antiviral, especialmente en las primeras etapas de la enfermedad. Se trata de una revisión integradora de la literatura, utilizando una metodología exploratoria y descriptiva. Los datos fueron organizados con el fin de enumerar los estudios relacionados con la temática en cuestión, el filtrado de los artículos encontrados tuvo como criterios de inclusión, artículos en los años 2020 y 2021 (excepto uno de ellos, en el año 2015), en el idiomas: portugués, inglés y español. Considerando que el SARS-CoV-2 suprime la expresión de IFN-I y los genes inducidos por IFN, interrumpiendo los mecanismos de defensa inmunológica, existe una relación de supresión de IFN con el empeoramiento del cuadro clínico en pacientes con COVID- 19. Con esto, la literatura aclara que la administración exógena de IFN-I ha demostrado reducir la severidad de los síntomas, sin embargo, este mecanismo necesita ser analizado con mayor claridad.

Palabras clave: Antivirales; Coronavirus; Interferones.

\section{Introdução}

O vírus SARS-CoV-2, causador da doença COVID-19, foi descrito pela primeira vez em dezembro de 2019, na cidade de Wuhan, China, e, em pouco tempo, obteve dimensões globais, já tendo infectado mais de 170 milhões de pessoas e causado mais de 3,5 milhões de mortes no mundo (World Health Organization, 2021), evoluindo desde uma leve gripe a comprometimentos respiratórios mais severos e falência múltipla de órgãos. Sugere-se que a infecção pelo vírus SARS-CoV-2 seja classificada em três diferentes estágios: a fase inicial da infecção, delimitada por tosse seca, febre e dor de cabeça, a fase pulmonar, delimitada pela dificuldade respiratória com ou sem hipoxemia e alterações na imaginologia pulmonar, a fase hiperinflamatória, delimitada pela tempestade de citocinas, IRHA (insuficiência respiratória hipoxemica aguda), IRA (insuficiência respiratória aguda) e disfunção orgânica múltipla (Siddiqi \& Mehra, 2020). Vários ensaios clínicos estão em andamento, porém, não se têm evidências que comprovem o uso de uma forma consistente e significativamente eficaz de tratamento medicamentoso para o controle do SARS-CoV-2 (World Health Organization, 2021), que surgiu como um grande desafio para a medicina atual, dominando os sistemas de saúde pública pelo mundo todo. Outra preocupação evidente é com o custo da terapia, que se agrava mais quando é considerado que cerca de $55 \%$ dos pacientes apresentam sintomas leves e moderados na fase inicial da infecção e $10 \%$ dos sintomas graves na fase pulmonar, existindo a possibilidade dos pacientes 
transitarem entre diferentes estágios da doença e aumentar consideravelmente o tempo de permanência hospitalar (Lima, 2020).

Vários estudos avaliaram a eficácia de algumas terapêuticas no tratamento da doença, por meio da testagem de alguns fármacos, como, Ribavirina, Remdesivir, Liponavir/Ritonavir e a própria Hidroxicloroquina, no qual seu uso gerou um amplo debate no Brasil, e no mundo, consequente aos questionamentos originados em relação à sua efetividade, assim como os outros medicamentos, que acabaram não demonstraram benefícios significativo (Ferreira, et al., 2020).

Em detrimento disso, a imunidade inata e a adaptativa, são as duas vertentes essenciais do sistema imunológico humano. No curso de infecções microbianas, a imunidade inata é a primeira vertente a entrar em ação. No decorrer da infecção viral do SARS-CoV-2, após a implantação primária, replicação local e disseminação do vírus para células hospedeiras vizinhas suscetíveis, o sistema imunológico inato atua primeiro e inicia sinalização celular ativando várias cascatas que levam a produção e liberação de citocinas pró-inflamatórias (tempestade de citocinas), caracterizada na maioria das vezes por baixos níveis de interferons (IFNs) tipo I e III. Os IFNs estimulam a expressão de vários genes que contribuem para o deslocamento das células hospedeiras em direção a um estado antiviral, impedindo a disseminação e replicação secundária do vírus (Bakadia, et al., 2021).

Os IFN-I (IFN- $\alpha$, IFN- $\beta$, IFN- $\omega$, IFN- $\varepsilon$ et IFN-א) são produzidos essencialmente pelas células dendríticas plasmocitóides (Pdc), e apresentam um comportamento autócrino e parácrino, induzindo a expressão de genes induzidos por interferon (ISGs) por meio da sinalização JAK-STAT, também envolvida na resposta antiviral, e já os IFN-III (IFN- $\lambda 1$, IFN$\lambda 2$, IFN- $\lambda 2$, IFN- $\lambda 4$ ) são expressos por células mieloides e epiteliais especializadas, produzindo efeitos semelhantes aos IFNI. Esses dois tipos de IFNs estão envolvidos na resposta inata antiviral da maioria das células, enquanto o INF-II (IFN- $\gamma$ ) serve predominantemente como a molécula de comunicação entre as células especializadas do sistema imunológico (Bakadia, et al., 2021). Com a consequente diminuição da expressão de IFN-I e/ou genes induzidos por IFN como demonstrado na figura 1, que acabam impedindo a prontidão das respostas imunes inatas e adaptativas. O SARS-CoV-2 suprime a expressão de IFN-I e genes induzidos por IFN, interrompendo assim as respostas imunes inatas e por consequência minimiza a ativação das respostas imunes adaptativas do sistema imunológico, que contribuem para a diminutiva da patogênese viral (Darazam, et al., 2021)

Em condições normais, quando o SARS-CoV-2 entra nas células humanas, ele se dirige ao endossomo, organela responsável pelo acoplamento dos receptores “Toll-like”, como TLR7 e TLR3, que fazem o reconhecimento RNA viral. Esta identificação conduz a uma via que leva à expressão de genes que codificam proteínas de IFN-I (Meffre \& Iwasaki, 2020). Como já foi citado que no decurso da SARS-CoV-2, a expressão e, subsequentemente, as funções dos IFN-I são relativamente suprimidas, a administração de IFN-I exógenos mostra reduzir a gravidade dos sintomas da doença. Vários estudos indicaram que IFN- $\beta$ é mais potente do que IFN- $\alpha$ como um inibidor do SARS-CoV-2, e, além disso, o Interferon Beta-1b (IFN $\beta 1 b$ ) e Interferon Beta-1a (IFN $\beta 1$ a), demonstraram ter os efeitos inibitórios mais potentes sobre o SARS-CoV-2 (Darazam, et al., 2021) Esse estudo tem como objetivo avaliar o potencial terapêutico do uso do Interferon na ativação do estado antiviral, principalmente nas fases iniciais da doença, contribuindo para a diminuição das complicações graves da Covid 19.

\section{Metodologia}

O presente estudo trata-se de uma revisão integrativa da literatura, que se utiliza de uma metodologia exploratória e descritiva (Pereira, et al., 2018).

Inicialmente, foram pesquisados estudos nas bases de dados eletrônicas: Literatura Latino-Americana e do Caribe em Ciências da Saúde (Lilacs); Scientific Electronic Library Online (SciELO) e Medical Literature Analysis and Retrieval System 
Online (Medline). Para pesquisa de tais artigos foram utilizados os descritores: antivirais; coronavírus; interferons. Na catalogação desses estudos usamos o operador booleano "AND" nos descritores das ciências da saúde (DECS).

Os dados foram organizados durante a revisão de literatura de forma a elencar os estudos relacionados à temática em questão, a filtragem dos artigos encontrados teve como critério de inclusão, artigos nos anos de 2020 e 2021 (com exceção de um deles, no ano de 2015), nos idiomas: português, inglês e espanhol, e como critério de exclusão artigos em outros idiomas e textos com apenas o resumo disponível. Após esse filtro ficou disponível 20 artigos para revisão. Para melhor compreensão, os métodos foram esquematizados na Figura 1. A distribuição dos dados da pesquisa por ano e base de dados estão apresentados no Quadro 1.

Figura 1 - Fluxograma esquematizando a metodologia do estudo.

\section{Revisão de literatura}

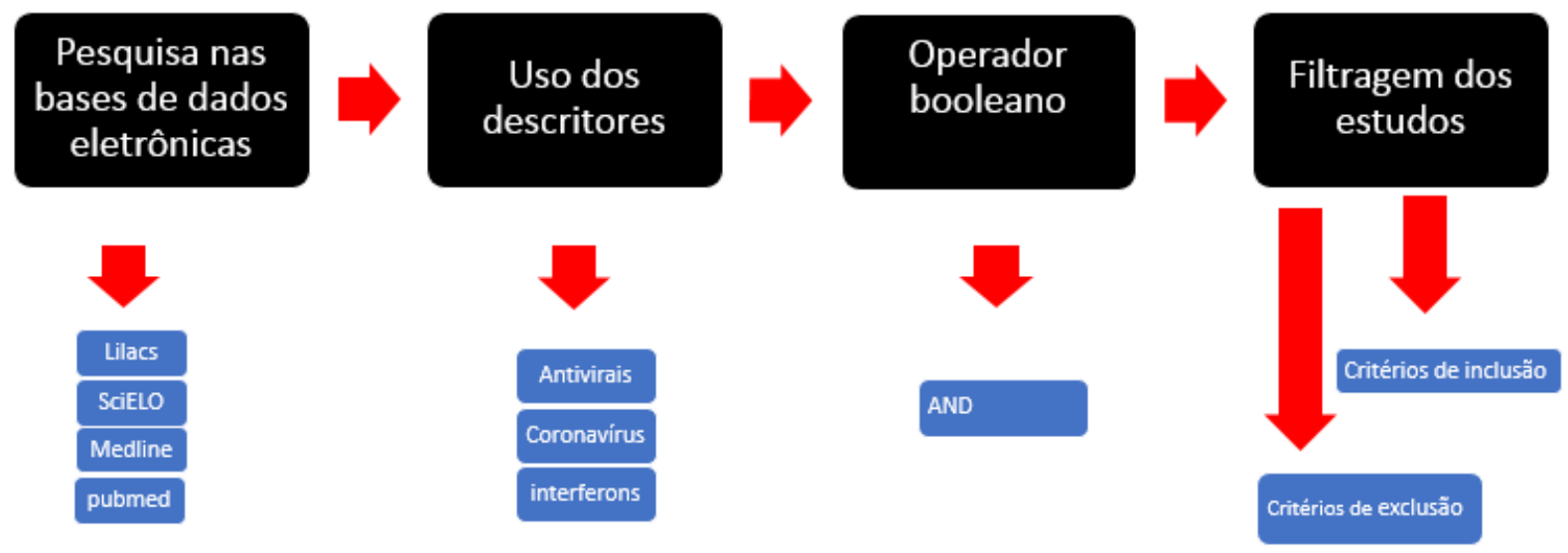

Fonte: Autores.

Quadro 1 - Distribuição dos dados da pesquisa em relação à base de dados e ano da publicação.

\begin{tabular}{|c|c|c|}
\hline Artigo & Base de dados & Ano de publicação \\
\hline $\begin{array}{l}\text { Bagheri, A., Moezzi, S., Mosaddeghi, P., Nadimi Parashkouhi, S., Fazel Hoseini, S. M., } \\
\text { Badakhshan, F., \& Negahdaripour, M. (2021). Interferon-inducer antivirals: Potential } \\
\text { candidates to combat COVID-19. International immunopharmacology, } 91,107245 . \\
\text { https://doi.org/10.1016/j.intimp.2020.107245 }\end{array}$ & PubMed & 2020 \\
\hline $\begin{array}{l}\text { Bakadia, B. M., He, F., Souho, T., Lamboni, L., Ullah, M. W., Boni, B. O., Ahmed, A., } \\
\text { Mukole, B. M., \& Yang, G. (2021). Prevention and treatment of COVID-19: Focus on } \\
\text { interferons, chloroquine/hydroxychloroquine, azithromycin, and vaccine. Biomedicine \& } \\
\text { pharmacotherapy }=\quad \text { Biomedecine } \quad \& \quad \text { pharmacotherapie, 133, } 111008 . \\
\text { https://doi.org/10.1016/j.biopha.2020.111008 }\end{array}$ & PubMed & 2020 \\
\hline $\begin{array}{l}\text { Busnadiego, I., Fernbach, S., Pohl, M. O., Karakus, U., Huber, M., Trkola, A., Stertz, S., \& } \\
\text { Hale, B. G. (2020). Antiviral Activity of Type I, II, and III Interferons Counterbalances } \\
\text { ACE2 Inducibility and Restricts SARS-CoV-2. mBio, 11(5), e01928-20. } \\
\text { https://doi.org/10.1128/mBio.01928-20 }\end{array}$ & PubMed & 2020 \\
\hline $\begin{array}{l}\text { Calabrese, LH, Winthrop, K., Strand, V., Yazdany, J., \& Walter, JE (2021). Interferon tipo } \\
\text { I, anticorpos anti-interferon e COVID-19. The Lancet Rheumatology , } 3 \text { (4), e246-e247. }\end{array}$ & PubMed & 2021 \\
\hline
\end{tabular}




\begin{tabular}{|c|c|c|}
\hline $\begin{array}{l}\text { Chan, J. F., Lau, S. K., To, K. K., Cheng, V. C., Woo, P. C., \& Yuen, K. Y. (2015). Middle } \\
\text { East respiratory syndrome coronavirus: another zoonotic betacoronavirus causing SARS- } \\
\text { like disease. Clinical microbiology reviews, } 28(2), 465 .\end{array}$ & Medline & 2015 \\
\hline $\begin{array}{l}\text { Felgenhauer, U., Schoen, A., Gad, H. H., Hartmann, R., Schaubmar, A. R., Failing, K., } \\
\text { Drosten, C., \& Weber, F. (2020). Inhibition of SARS-CoV-2 by type I and type III } \\
\text { interferons. The Journal of biological chemistry, 295(41), 13958-13964. } \\
\text { https://doi.org/10.1074/jbc.AC120.013788 }\end{array}$ & PubMed & 2020 \\
\hline $\begin{array}{l}\text { Hung, I. F., Lung, K. C., Tso, E. Y., Liu, R., Chung, T. W., Chu, M. Y., Ng, Y. Y., Lo, J., } \\
\text { Chan, J., Tam, A. R., Shum, H. P., Chan, V., Wu, A. K., Sin, K. M., Leung, W. S., Law, W. } \\
\text { L., Lung, D. C., Sin, S., Yeung, P., Yip, C. C., ... Yuen, K. Y. (2020). Triple combination of } \\
\text { interferon beta-1b, lopinavir-ritonavir, and ribavirin in the treatment of patients admitted } \\
\text { to hospital with COVID-19: an open-label, randomised, phase } 2 \text { trial. Lancet (London, } \\
\text { England), 395(10238), 1695-1704. https://doi.org/10.1016/S0140-6736(20)31042-4 }\end{array}$ & Lilacs & 2020 \\
\hline $\begin{array}{l}\text { Khamis, F., Al Naabi, H., Al Lawati, A., Ambusaidi, Z., Al Sharji, M., Al Barwani, U., } \\
\text { Pandak, N., Al Balushi, Z., Al Bahrani, M., Al Salmi, I., \& Al-Zakwani, I. (2021). } \\
\text { Randomized controlled open label trial on the use of favipiravir combined with inhaled } \\
\text { interferon beta-1b in hospitalized patients with moderate to severe COVID-19 } \\
\text { pneumonia. International journal of infectious diseases: IJID: official publication of the } \\
\text { International Society for Infectious } \text { Diseases, 102, } 538-543 . \\
\text { https://doi.org/10.1016/j.ijid.2020.11.008 }\end{array}$ & PubMed & 2021 \\
\hline Lima, C. M. A. D. O. (2020). Informações sobre o novo coronavírus (COVID-19). & Scielo & 2020 \\
\hline $\begin{array}{l}\text { Malekzadeh, R., Murthy, S., Reddy, K. S., Roses Periago, M., Abi Hanna, P., Ader, F., Al- } \\
\text { Bader, A. M., Alhasawi, A., Allum, E., Alotaibi, A., ... Swaminathan, S. (2021). Repurposed } \\
\text { Antiviral Drugs for Covid-19 - Interim WHO Solidarity Trial Results. The New England } \\
\text { journal of medicine, 384(6), 497-511. } \underline{\text { https://doi.org/10.1056/NEJMoa2023184 }}\end{array}$ & PubMed & 2021 \\
\hline $\begin{array}{l}\text { Mantlo, E., Bukreyeva, N., Maruyama, J., Paessler, S., \& Huang, C. (2020). Antiviral } \\
\text { activities of type I interferons to SARS-CoV-2 infection. Antiviral research, 179, } 104811 . \\
\text { https://doi.org/10.1016/j.antiviral.2020.104811 }\end{array}$ & PubMed & 2020 \\
\hline $\begin{array}{l}\text { MEDEIROS-PESQUISADORA, Flávia Cordeiro et al. INTERFERON } \\
\text { PROFALAXIA E TRATAMENTO DE COVID-19. Revisão sistemática rápida. }\end{array}$ & Scielo & 2020 \\
\hline $\begin{array}{l}\text { Meffre E, Iwasaki A. Interferon deficiency can lead to severe COVID. Nature. } 2020 \\
\text { Nov;587(7834):374-376. doi: 10.1038/d41586-020-03070-1. PMID: 33139913. }\end{array}$ & PubMed & 2020 \\
\hline $\begin{array}{l}\text { Monk, P. D., Marsden, R. J., Tear, V. J., Brookes, J., Batten, T. N., Mankowski, M., } \\
\text { Gabbay, F. J., Davies, D. E., Holgate, S. T., Ho, L. P., Clark, T., Djukanovic, R., Wilkinson, } \\
\text { T., \& Inhaled Interferon Beta COVID-19 Study Group (2021). Safety and efficacy of inhaled } \\
\text { nebulised interferon beta-1a (SNG001) for treatment of SARS-CoV-2 infection: a } \\
\text { randomised, double-blind, placebo-controlled, phase } 2 \text { trial. The Lancet. Respiratory } \\
\text { medicine, 9(2), 196-206. https://doi.org/10.1016/S2213-2600(20)30511-7 }\end{array}$ & PubMed & 2021 \\
\hline $\begin{array}{l}\text { Nile, S. H., Nile, A., Qiu, J., Li, L., Jia, X., \& Kai, G. (2020). COVID-19: Pathogenesis, } \\
\text { cytokine storm and therapeutic potential of interferons. Cytokine \& growth factor } \\
\text { reviews, 53, 66-70. https://doi.org/10.1016/j.cytogfr.2020.05.002 }\end{array}$ & PubMed & 2020 \\
\hline $\begin{array}{l}\text { Park, A., \& Iwasaki, A. (2020). Type I and Type III Interferons - Induction, Signaling, } \\
\text { Evasion, and Application to Combat COVID-19. Cell host \& microbe, 27(6), 870-878. } \\
\text { https://doi.org/10.1016/j.chom.2020.05.008 }\end{array}$ & PubMed & 2020 \\
\hline $\begin{array}{l}\text { Peiffer-Smadja, N., \& Yazdanpanah, Y. (2021). Interferon beta-1a nebulizado para } \\
\text { pacientes com COVID-19. The Lancet Respiratory Medicine }, 9(2), 122-123 \text {. }\end{array}$ & Scielo & 2021 \\
\hline
\end{tabular}




\begin{tabular}{|c|c|c|}
\hline $\begin{array}{l}\text { Pumapillo Garcia, A. S., \& Quispe Castillo, C. Z. (2021). Esquema de manejo de COVID-19 } \\
\text { en adultos. Horizonte Médico (Lima), 21(1). }\end{array}$ & Lilacs & 2021 \\
\hline $\begin{array}{l}\text { Pumapillo Garcia, Ana Sarai, \& Quispe Castillo, Carmen Zaira. (2021). Esquema de } \\
\text { manejo de COVID-19 en adultos. Horizonte Médico (Lima), 21(1), e1362. } \\
\text { https://dx.doi.org/10.24265/horizmed.2021.v21n1.11 }\end{array}$ & PubMed & 2021 \\
\hline $\begin{array}{l}\text { Rahmani, H., Davoudi-Monfared, E., Nourian, A., Khalili, H., Hajizadeh, N., Jalalabadi, N. } \\
\text { Z., Fazeli, M. R., Ghazaeian, M., \& Yekaninejad, M. S. (2020). Interferon } \beta \text {-1b in treatment } \\
\text { of severe COVID-19: A randomized clinical trial. International immunopharmacology, 88, } \\
\text { 106903. https://doi.org/10.1016/j.intimp.2020.106903 }\end{array}$ & PubMed & 2020 \\
\hline $\begin{array}{l}\text { Siddiqi, H. K., \& Mehra, M. R. (2020). COVID-19 illness in native and immunosuppressed } \\
\text { states: A clinical-therapeutic staging proposal. The journal of heart and lung } \\
\text { transplantation, 39(5), } 405 .\end{array}$ & Pubmed & 2020 \\
\hline $\begin{array}{l}\text { Xavier, AR, Silva, JS, Almeida, JPC, Conceição, JFF, Lacerda, GS, \& Kanaan, S. } \\
\text { (2020). COVID-19: manifestações clínicas e laboratoriais na infecção pelo novo } \\
\text { coronavírus. Jornal Brasileiro de Patologia e Medicina Laboratorial, 56. }\end{array}$ & Scielo & 2020 \\
\hline $\begin{array}{l}\text { Yang, L., Xie, X., Tu, Z., Fu, J., Xu, D., \& Zhou, Y. (2021). The signal pathways and } \\
\text { treatment of cytokine storm in COVID-19. Signal transduction and targeted therapy, 6(1), } \\
\text { 255. https://doi.org/10.1038/s41392-021-00679-0 }\end{array}$ & PubMed & 2021 \\
\hline
\end{tabular}

Fonte: Autores.

\section{Resultados e Discussão}

Em casos de infecções causadas pelo Sars-CoV-2, as possíveis opções terapêuticas em estudo atualmente incluem: inibidores de enzimas, nucleosídeos, agentes alvos de hospedeiro, plasma convalescente e os interferons (IFNs). Os IFNs utilizados terapeuticamente são produzidos com base na tecnologia de DNA recombinante, e são eles: IFN $\alpha$-2a (Roferon), IFN $\alpha$-2b (Intron A), IFN $\alpha$-n1 (Wellferon), IFN $\alpha$-n3 (Alferon), IFN $\alpha$-con 1 (In IFN $\beta$-1a (Rebif), IFN $\beta$-1b (Betaferon), IFN $\beta$ 1a (Avonex), IFN $\beta-1 b$ (Betaseron), IFN $\alpha-2 a$ (Pegasys), IFN $\alpha-2 b$ (PegIntron), IFN $\alpha$ P-2b (Sylatron) e IFN $\gamma$-1b (Acimmune) que estão disponíveis clinicamente. Há indícos que o tratamento com IFN $-\alpha$, IFN $-\beta$ e IFNs exógenos são eficazes na inibição da síntese proteica e na replicação dos vírus SARS-CoV-2, SARS-CoV e MERS-CoV (Nile, et al., 2020). Além disso, a utilização dos IFNs em combinação com outros medicamentos pode diminuir os efeitos adversos causados pela infecção por SARS-CoV-2, como a tempestade de citocinas inflamatórias, além de melhorar a função pulmonar e reduzir a carga viral (Nile, et al., 2020).

Os principais IFNs que podem ser utilizados para o tratamento contra o SARS-CoV-2, são os do tipo I (IFN- $\alpha$, IFN$\beta$ ), que possuem potencial para inibir a replicação do vírus e consequentemente melhorar os resultados clínicos (Khamis, el al., 2021). Por exemplo, a administração precoce do IFN- $\alpha 2 b$ inalado foi associada à redução da mortalidade e menor permanência hospitalar em pacientes (Bagheri, et al., 2021). No caso da utilização do IFN $\beta$-1a, percebeu-se desfechos clínicos com padrão benéfico quando inalado como nebulização em pacientes internados com Covid-19, contudo não houve diferença significativa nas chances de alta hospitalar ou no tempo necessário para isso (Monk, et al., 2021). Nesse sentido, quanto ao IFN $\beta-1 b$, notou-se que ele também possui bom grau de seguridade, e pode ser eficaz no tratamento contra o SARS-CoV-2 em estágios iniciais da infecção, mas não em estágios avançados (Khamis, el al., 2021). 
Figura 1 - Via de sinalização antiviral defeituosa. Foi descoberto que pessoas que desenvolvem Covid-19 grave possuem mutações nos genes que codificam proteínas de IFN-I (em vermelho), e que, por tanto, apresentam falhas na produção de interferon. Mutações nos genes que codificam receptores de interferon (proteínas IFNAR1 e IFNAR2). Também há relatos de mutações genéticas específicas em pacientes com Covid-19 grave, as quais produzem autoanticorpos contra os IFN-I, IFN- $\alpha$ e IFN- $\omega$, bloqueando a sinalização mediada por esses interferons, o que impede a expressão do gene antiviral.

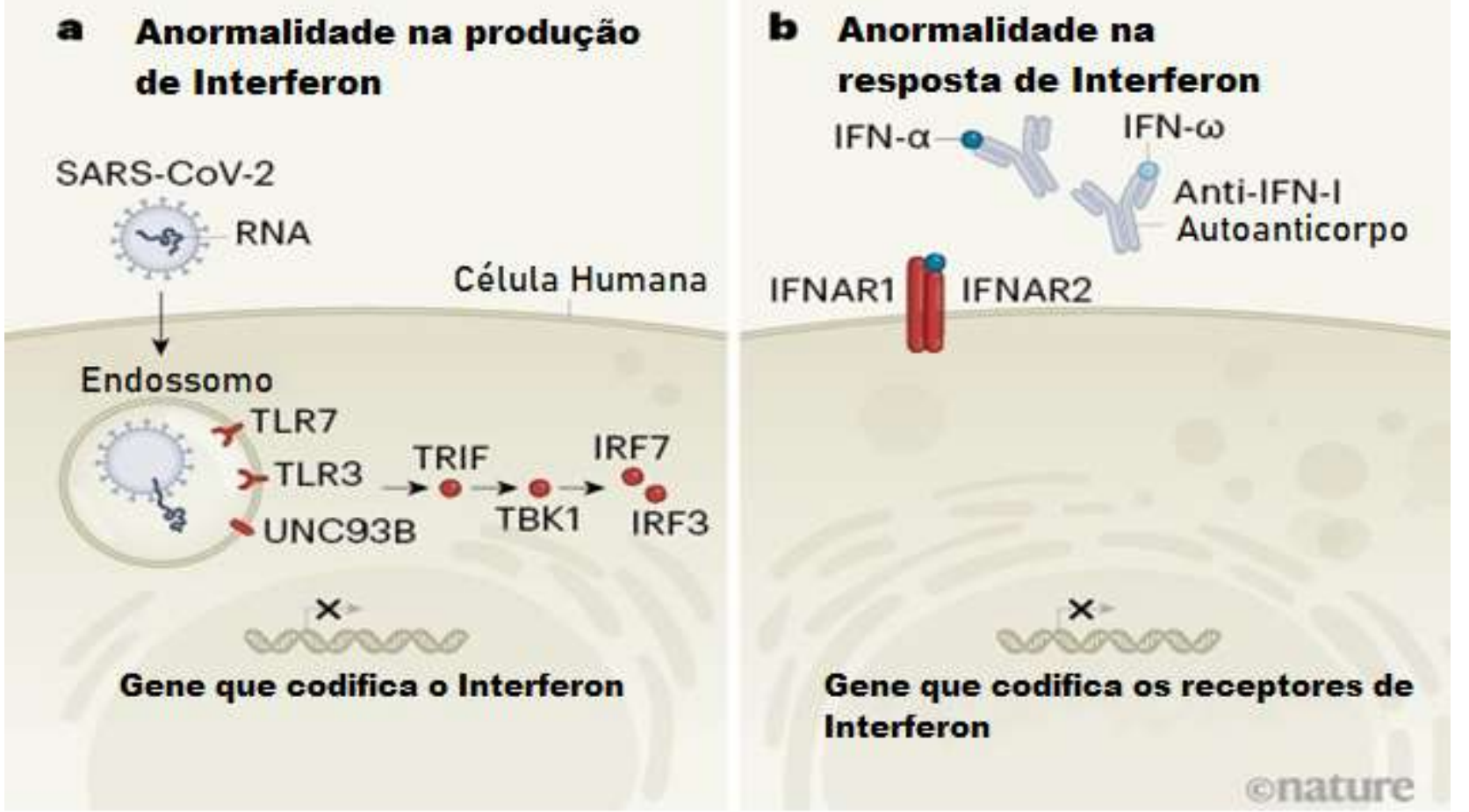

Fonte: Adaptado de Meffre e Iwasaki (2020).

Aparentemente, o IFN $\beta$-1b como terapia complementar encurta significativamente o tempo de resposta clínica, aumenta a taxa de alta e diminui a necessidade de internação na UTI nesses pacientes. Ou seja, quando iniciado o tratamento com IFN $\beta-1 b$ de forma precoce, as chances de desenvolvimento de efeitos adversos graves decorrentes da Covid-19 acabam diminuindo (Rahmani, et al., 2020). O uso de IFN-I já é estudado há algum tempo para o tratamento de infecções causadas por outros coronavírus (MERS-CoV e SARS-CoV), e nisso, percebeu-se que, eles apresentavam um potencial positivo na imunidade inata contra essas infecções (Chan, et al., 2015). E, por tanto, pode haver um benefício para o tratamento da doença causada pelo SARS-CoV-2.

Os tratamentos realizados com combinação medicamentosa também possuem seu potencial terapêutico no tratamento da Covid-19, por exemplo, a associação de IFN $\beta$-1b injetável, com lopinavir-ritonavir e ribavirina para o tratamento de pacientes internados com Covid-19 é significativamente mais segura e mais efetiva quanto ao quadro clínico da infecção do que em alguns casos em que eles são utilizados de forma isolada. Contudo, os pacientes podem apresentar eventos adversos como, diarreia, febre, náuseas e em virtude da limitação desses estudos, a verdadeira eficácia desse tratamento ainda está em análise (Hung, et al., 2020).

Embora a administração de IFNs seja uma estratégia de tratamento globalmente aceita para estimular as respostas imunes antivirais, a terapia com IFNs para a Covid-19 se apresenta bastante controverso. Aparentemente, o uso dos IFNs em estágios iniciais da infecção produz resultados efetivos, mas essa estratégia se mostra prejudicial nos estágios graves ou posteriores da infecção (Bagheri, et al. 2021). Além disso, considerou-se também o potencial do uso dos IFNs (IFN $\alpha$-1b, principalmente) como tratamento profilático, contudo, os atuais estudos ainda não confirmam essa possibilidade (Medeiros, 
2020). Algumas controvérsias presentes nos estudos podem ser explicadas pela falta de padronização quanto à realização de estudos e na seleção dos grupos a serem estudados (Peiffer-Smadja, 2021), por isso, a referida pesquisa toma-se relevante para o meio científico a fim de se ter uma melhor compreensão sobre a real eficácia desse tratamento utilizando IFNs contra a Covid-19. A distribuição dos dados de acordo com o tipo de estudo, grupo alvo, tipo de tratamento e resultados são demonstrados no Quadro 2.

Quadro 2 - Distribuição dos dados de pesquisa de acordo com o tipo de estudo, grupo alvo, tipo de tratamento e resultados.

\begin{tabular}{|c|c|c|c|c|c|c|c|}
\hline $\begin{array}{l}\text { AUTOR/ } \\
\text { ANO }\end{array}$ & $\mathbf{n}$ & ESTUDO & GRUPO & TRATAMENTO & CONTROLE & TESTE & RESULTADOS \\
\hline $\begin{array}{l}\text { Khamis et al. } \\
(2021)\end{array}$ & 89 & $\begin{array}{l}\text { Ensaio } \\
\text { Randomizado } \\
\text { controlado } \\
\text { por rótulo } \\
\text { aberto. }\end{array}$ & $\begin{array}{l}\text { Adultos } \\
\text { internados } \\
\text { com } \\
\text { pneumonia } \\
\text { decorrente de } \\
\text { Covid-19. }\end{array}$ & $\begin{array}{l}\text { Favipiravir } \text { oral } \\
\text { associado à } \\
\beta-1 \mathrm{~b} \text { inalado }\end{array}$ & $\begin{array}{l}\text { Hidroxicloroquina } \\
\text { (HCQ). } \mathrm{n}=45\end{array}$ & $\begin{array}{l}\text { Favipiravir } \\
\text { com IFN } \beta \text { - } \\
\text { 1b. } n=44\end{array}$ & $\begin{array}{l}\text { Não foi } \\
\text { percebido } \\
\text { diferenças nos } \\
\text { desfechos } \\
\text { clínicos entre os } \\
\text { medicamentos } \\
\text { testados. } \\
\end{array}$ \\
\hline $\begin{array}{l}\text { Hung et al. } \\
(\mathbf{2 0 2 0})\end{array}$ & 127 & $\begin{array}{l}\text { Ensaio de } \\
\text { marca aberta, } \\
\text { randomizado, } \\
\text { fase } 2 . \\
\text { Prospectivo }\end{array}$ & $\begin{array}{l}\text { Adultos com } \\
\text { COVID-19 } \\
\text { que foram } \\
\text { admitidos em } \\
\text { seis hospitais } \\
\text { de Hong Kong }\end{array}$ & $\begin{array}{l}\text { Combinação } \\
\text { tripla de IFN } \beta \text { - } \\
1 \mathrm{~b}, \quad \text { lopinavir- } \\
\text { ritonavir } \\
\text { ribavirina. }\end{array}$ & $\begin{array}{l}\text { Lopinavir } \\
\text { ritonavir. } \mathrm{n}=86\end{array}$ & $\begin{array}{l}\text { Combinação } \\
\text { tripla. } \mathrm{n}=41\end{array}$ & $\begin{array}{l}\text { O uso da } \\
\text { combinação } \\
\text { tripla foi segura } \\
\text { e superior ao } \\
\text { grupo controle } \\
\text { quanto ao alívio } \\
\text { dos sintomas e } \\
\text { no encurtamento } \\
\text { do tempo de } \\
\text { eliminação do } \\
\text { vírus. }\end{array}$ \\
\hline $\begin{array}{l}\text { Monk } \\
(2021)\end{array}$ & 101 & $\begin{array}{l}\text { Teste duplo- } \\
\text { cego } \\
\text { randomizado, } \\
\text { fase } 2 \text {. }\end{array}$ & $\begin{array}{l}\text { Adultos com } \\
18 \text { anos ou } \\
\text { mais } \\
\text { internados no } \\
\text { hospital com } \\
\text { sintomas } \\
\text { COVID-19, } \\
\text { com RT-PCR } \\
\text { ou teste de } \\
\text { ponto de } \\
\text { atendimento } \\
\text { positivos. }\end{array}$ & $\begin{array}{l}\text { Inalação de IFN } \\
\beta-1 \text { a nebulizado } \\
\text { (SNG001) }\end{array}$ & Placebo. $\mathrm{n}=51$ & $\begin{array}{l}\text { SNG001. } \\
\mathrm{n}=50\end{array}$ & $\begin{array}{l}\text { Os pacientes que } \\
\text { receberam } \\
\text { SNG001 } \\
\text { tiveram maiores } \\
\text { chances de } \\
\text { melhora e se } \\
\text { recuperaram } \\
\text { mais } \\
\text { rapidamente da } \\
\text { infecção pelo } \\
\text { SARS-CoV-2 } \\
\text { do que os } \\
\text { pacientes que } \\
\text { receberam } \\
\text { placebo. }\end{array}$ \\
\hline $\begin{array}{l}\text { Pandit et al. } \\
(2021)\end{array}$ & 40 & $\begin{array}{l}\text { Ensaio } \\
\text { randomizado } \\
\text { de rótulo } \\
\text { aberto, fase } 2 .\end{array}$ & $\begin{array}{l}\text { Adultos com } \\
\text { idade } \geq 18 \\
\text { anos com RT- } \\
\text { PCR } \\
\text { confirmado } \\
\text { para COVID- } \\
19 \text { e sintomas } \\
\text { moderados. }\end{array}$ & $\begin{array}{l}\text { PEG-IFN- } \alpha 2 b \\
\text { juntamente com o } \\
\text { padrão de } \\
\text { cuidado (SOC). }\end{array}$ & $\begin{array}{l}\text { Apenas SOC. } \mathrm{n}= \\
20\end{array}$ & $\begin{array}{l}\text { PEG-IFN- } \\
\alpha 2 \mathrm{~b} \\
\text { juntamente } \\
\text { com SOC. } \mathrm{n} \\
=20\end{array}$ & $\begin{array}{l}\text { Os pacientes que } \\
\text { receberam o } \\
\text { tratamento com } \\
\text { PEG-IFN- } \alpha 2 b \\
\text { juntamente com } \\
\text { SOC tiveram } \\
\text { melhora } \\
\text { significativa do } \\
\text { estado clínico e } \\
\text { na redução viral. }\end{array}$ \\
\hline $\begin{array}{l}\text { Darazam et al. } \\
\text { (2021) }\end{array}$ & 60 & $\begin{array}{l}\text { Ensaio } \\
\text { controlado } \\
\text { randomizado } \\
\text { triplo de } \\
\text { rótulo aberto }\end{array}$ & $\begin{array}{l}\text { Pacientes com } \\
\text { idade } \geq 18 \\
\text { anos } \\
\text { gravemente } \\
\text { doentes, com } \\
\text { RT-PCR } \\
\text { positivo e } \\
\text { tomografias } \\
\text { torácicas } \\
\text { indicadoras. }\end{array}$ & $\begin{array}{l}\text { IFN } \beta \text {-1a ou IFN } \\
\beta \text {-1b associados a } \\
\text { lopinavir- } \\
\text { ritonavir oral e } \\
\text { HCQ. }\end{array}$ & $\begin{array}{l}\text { Apenas lopinavir- } \\
\text { ritonavir oral e } \\
\text { HCQ. } n=20\end{array}$ & $\begin{array}{l}\text { Adição de } \\
\text { IFN } \beta-1 a . n \\
=20 \\
\text { e } \\
\text { Adição de } \\
\text { IFN } \beta \text {-1b. n } \\
=20\end{array}$ & $\begin{array}{l}\text { A mortalidade } \\
\text { foi } \\
\text { numericamente } \\
\text { menor em } \\
\text { ambos os grupos } \\
\text { teste. Não houve } \\
\text { diferenças } \\
\text { significativas } \\
\text { entre os três } \\
\text { ramos } \quad \text { em } \\
\text { relação aos }\end{array}$ \\
\hline
\end{tabular}




\begin{tabular}{|c|c|c|c|c|c|c|}
\hline & & & & & & $\begin{array}{l}\text { eventos } \\
\text { adversos. }\end{array}$ \\
\hline Li el al. (2021) & 94 & $\begin{array}{l}\text { Ensaio } \\
\text { randomizado } \\
\text { multicêntrico. }\end{array}$ & $\begin{array}{l}\text { Pacientes } \\
\text { hospitalizados } \\
\text { com COVID- } \\
19 \text { moderada a } \\
\text { grave. }\end{array}$ & $\begin{array}{l}\text { Nebulização com IFN- } \alpha . \mathrm{n}=48 \\
\text { novo interferon } \\
\text { supercomposto } \\
\text { recombinante } \\
\text { geneticamente } \\
\text { modificado } \\
\text { (rSIFN-co) ou } \\
\text { nebulização com } \\
\text { IFN- } \alpha \text {. Cada um } \\
\text { associado aos } \\
\text { antivirais de linha } \\
\text { de base. }\end{array}$ & $\begin{array}{l}\text { rSIFN-co. } \mathrm{n} \\
=46\end{array}$ & $\begin{array}{l}\text { SIFN-co foi } \\
\text { associado a um } \\
\text { menor tempo de } \\
\text { melhora clínica } \\
\text { do que o IFN- } \alpha \\
\text { no tratamento de } \\
\text { COVID-19 } \\
\text { moderado a } \\
\text { grave quando } \\
\text { combinado com } \\
\text { agentes } \\
\text { antivirais de } \\
\text { linha de base. }\end{array}$ \\
\hline
\end{tabular}

Fonte: Autores.

\section{Conclusão}

Considerando que o SARS-CoV-2 suprime a expressão do IFN-I e os genes induzidos por IFN, de modo a interromper os mecanismos de defesa do sistema imunológico contribuintes para abrandar os efeitos do vírus no organismo e as mutações genéticas descritas em pacientes que desenvolvem a forma grave da infecção, ligadas à produção de autoanticorpos contra o IFN-I, IFN- $\alpha$ e IFN- $\omega$, nota-se uma estreita relação da supressão dos IFN com o agravamento do quadro clínico em pacientes com COVID-19. Com base nisso, a literatura elucida que a administração exógena de IFN-I mostra reduzir a gravidade dos sintomas da doença, porém, esse mecanismo precisa ser analisado com maior clareza. Ademais, de acordo com os estudos citados anteriormente e por meio de análises dos índices encontrados, pode-se inferir que a terapia mediada por interferon pode contribuir para amenizar as complicações associadas à COVID-19, uma vez que, por meio da formação do complexo imune, proporciona a ativação do estado antiviral. Todavia, até o presente momento a segurança relacionada à profilaxia e eficácia dessa possível terapêutica contra o vírus SARS-CoV-2 é considerada limitada, necessitando ser mais bem compreendida, de modo a esclarecer a real eficácia e as consequências do uso do interferon no tratamento da infecção.

Trabalhos futuros são necessários para avaliar a segurança e eficácia da administração exógena de IFN-I sozinho ou em adição a outros medicamentos na redução da mortalidade e no combate à pandemia da COVID-19.

\section{Referências}

Bagheri, A., Moezzi, S., Mosaddeghi, P., Nadimi Parashkouhi, S., Fazel Hoseini, S. M., Badakhshan, F., \& Negahdaripour, M. (2021). Interferon-inducer antivirals: Potential candidates to combat COVID-19. International immunopharmacology, 91, 107245. https://doi.org/10.1016/j.intimp.2020.107245

Bakadia, B. M., He, F., Souho, T., Lamboni, L., Ullah, M. W., Boni, B. O., Ahmed, A., Mukole, B. M., \& Yang, G. (2021). Prevention and treatment of COVID-19: Focus on interferons, chloroquine/hydroxychloroquine, azithromycin, and vaccine. Biomedicine \& pharmacotherapy = Biomedecine \& pharmacotherapie, 133, 111008. https://doi.org/10.1016/j.biopha.2020.111008

Busnadiego, I., Fernbach, S., Pohl, M. O., Karakus, U., Huber, M., Trkola, A., Stertz, S., \& Hale, B. G. (2020). Antiviral Activity of Type I, II, and III Interferons Counterbalances ACE2 Inducibility and Restricts SARS-CoV-2. mBio, 11(5), e01928-20. https://doi.org/10.1128/mBio.01928-20

Calabrese, LH, Winthrop, K., Strand, V., Yazdany, J., \& Walter, JE (2021). Interferon tipo I, anticorpos anti-interferon e COVID-19. The Lancet Rheumatology, 3 (4), e246-e247.

Chan, J. F., Lau, S. K., To, K. K., Cheng, V. C., Woo, P. C., \& Yuen, K. Y. (2015). Middle East respiratory syndrome coronavirus: another zoonotic betacoronavirus causing SARS-like disease. Clinical microbiology reviews, 28(2), 465.

Darazam, I. A., Shokouhi, S., Pourhoseingholi, M. A., Irvani, S. S. N., Mokhtari, M., Shabani, M., ... \& Khoshkar, A. (2021). Role of interferon therapy in severe COVID-19: the COVIFERON randomized controlled trial. Scientific reports, 11(1), 1-11.

Felgenhauer, U., Schoen, A., Gad, H. H., Hartmann, R., Schaubmar, A. R., Failing, K., Drosten, C., \& Weber, F. (2020). Inhibition of SARS-CoV-2 by type I and type III interferons. The Journal of biological chemistry, 295(41), 13958-13964. https://doi.org/10.1074/jbc.AC120.013788 
Hung, I. F., Lung, K. C., Tso, E. Y., Liu, R., Chung, T. W., Chu, M. Y., Ng, Y. Y., Lo, J., Chan, J., Tam, A. R., Shum, H. P., Chan, V., Wu, A. K., Sin, K. M., Leung, W. S., Law, W. L., Lung, D. C., Sin, S., Yeung, P., Yip, C. C., ... Yuen, K. Y. (2020). Triple combination of interferon beta-1b, lopinavir-ritonavir, and ribavirin in the treatment of patients admitted to hospital with COVID-19: an open-label, randomised, phase 2 trial. Lancet (London, England), 395(10238), 1695-1704. https://doi.org/10.1016/S0140-6736(20)31042-4

Khamis, F., Al Naabi, H., Al Lawati, A., Ambusaidi, Z., Al Sharji, M., Al Barwani, U., Pandak, N., Al Balushi, Z., Al Bahrani, M., Al Salmi, I., \& AlZakwani, I. (2021). Randomized controlled open label trial on the use of favipiravir combined with inhaled interferon beta-1b in hospitalized patients with moderate to severe COVID-19 pneumonia. International journal of infectious diseases : IJID : official publication of the International Society for Infectious

Diseases, 102, 538-543. https://doi.org/10.1016/j.ijid.2020.11.008

Lima, C. M. A. D. O. (2020). Informações sobre o novo coronavírus (COVID-19).

Malekzadeh, R., Murthy, S., Reddy, K. S., Roses Periago, M., Abi Hanna, P., Ader, F., Al-Bader, A. M., Alhasawi, A., Allum, E., Alotaibi, A., ... Swaminathan, S. (2021). Repurposed Antiviral Drugs for Covid-19 - Interim WHO Solidarity Trial Results. The New England journal of medicine, 384(6), 497-511. https://doi.org/10.1056/NEJMoa2023184

Mantlo, E., Bukreyeva, N., Maruyama, J., Paessler, S., \& Huang, C. (2020). Antiviral activities of type I interferons to SARS-CoV-2 infection. Antiviral research, 179, 104811. https://doi.org/10.1016/j.antiviral.2020.104811

Medeiros-Pesquisadora, F. C., et al. Interferon Para Profilaxia e Tratamento de Covid-19. Revisão sistemática rápida.

Meffre, E., \& Iwasaki, A. (2020). Interferon deficiency can lead to severe COVID.

Monk, P. D., Marsden, R. J., Tear, V. J., Brookes, J., Batten, T. N., Mankowski, M., Gabbay, F. J., Davies, D. E., Holgate, S. T., Ho, L. P., Clark, T., Djukanovic, R., Wilkinson, T., \& Inhaled Interferon Beta COVID-19 Study Group (2021). Safety and efficacy of inhaled nebulised interferon beta-1a (SNG001) for treatment of SARS-CoV-2 infection: a randomised, double-blind, placebo-controlled, phase 2 trial. The Lancet. Respiratory medicine, 9(2), 196-206. https://doi.org/10.1016/S2213-2600(20)30511-7

Nile, S. H., Nile, A., Qiu, J., Li, L., Jia, X., \& Kai, G. (2020). COVID-19: Pathogenesis, cytokine storm and therapeutic potential of interferons. Cytokine \& growth factor reviews, 53, 66-70. https://doi.org/10.1016/j.cytogfr.2020.05.002

Park, A., \& Iwasaki, A. (2020). Type I and Type III Interferons - Induction, Signaling, Evasion, and Application to Combat COVID-19. Cell host \& microbe, 27(6), 870-878. https://doi.org/10.1016/j.chom.2020.05.008

Peiffer-Smadja, N., \& Yazdanpanah, Y. (2021). Interferon beta-1a nebulizado para pacientes com COVID-19. The Lancet Respiratory Medicine , 9 (2), $122-$ 123.

Pereira, A. S., Shitsuka, D. M., Parreira, F. J., \& Shitsuka, R. (2018). Metodologia da pesquisa científica.

Pumapillo Garcia, A. S., \& Quispe Castillo, C. Z. (2021). Esquema de manejo de COVID-19 en adultos. Horizonte Médico (Lima), 21(1).

Pumapillo Garcia, A. S., \& Quispe Castillo, C. Z. (2021). Esquema de manejo de COVID-19 en adultos. Horizonte Médico (Lima), $21(1)$, e1362. https://dx.doi.org/10.24265/horizmed.2021.v21n1.11

Rahmani, H., Davoudi-Monfared, E., Nourian, A., Khalili, H., Hajizadeh, N., Jalalabadi, N. Z., Fazeli, M. R., Ghazaeian, M., \& Yekaninejad, M. S. (2020). Interferon $\beta-1 \mathrm{~b}$ in treatment of severe COVID-19: A randomized clinical trial. International immunopharmacology, 88, 106903. https://doi.org/10.1016/j.intimp.2020.106903

Siddiqi, H. K., \& Mehra, M. R. (2020). COVID-19 illness in native and immunosuppressed states: A clinical-therapeutic staging proposal. The journal of heart and lung transplantation, 39(5), 405.

World Health Organization. (2021). Coronavirus disease ( COVID-19): weekly epidemiological update.

Xavier, A. R., Silva, J. S., Almeida, J. P. C., Conceição, J. F. F., Lacerda, G. S., \& Kanaan, S. (2020). COVID-19: manifestações clínicas e laboratoriais na infecção pelo novo coronavírus. Jornal Brasileiro de Patologia e Medicina Laboratorial, 56.

Yang, L., Xie, X., Tu, Z., Fu, J., Xu, D., \& Zhou, Y. (2021). The signal pathways and treatment of cytokine storm in COVID-19. Signal transduction and targeted therapy, 6(1), 255. https://doi.org/10.1038/s41392-021-00679-0 